\title{
FUNCTIONALIZATION OF 3D FIBROUS SCAFFOLDS PREPARED USING CENTRIFUGAL SPINNING WITH LIPOSOMES AS A SIMPLE DRUG DELIVERY SYSTEM
}

\author{
Michala Rampichová ${ }^{a, *}$, Matej Buzgo $^{a}$, V̌̌ra Lukášová ${ }^{b}$, \\ Andrea MíčKováa ${ }^{a, b}$, Karolí́na VocetKováa ${ }^{a, b}$, VĚra Sovková ${ }^{b}$, \\ FrANCO RUstichelli $^{b}$, EvŽEN AMLER ${ }^{a, b}$ \\ ${ }^{a}$ UCEEB, Czech Technical University in Prague, Bustehrad, Czech Republic \\ ${ }^{b}$ Institute of Experimental Medicine of CAS, Prague, Czech Republic \\ * corresponding author: michala.rampichova@uceeb.cz
}

\begin{abstract}
D materials supporting cell adhesion, infiltration and proliferation are crucial for bone tissue engineering. To fulfill these expectations, it is beneficial to combine the 3D scaffold with drug delivery systems. Release of the bioactive molecules directly in the site of the defect results in attraction of cells and their proliferation and differentiation in the required cell type. Only properly differentiated cells produce proteins of extracellular matrix and can form new tissue. In the current study, we propose a simple drug delivery system composed of polycaprolactone fibers, prepared using centrifugal spinning, with adhered liposomes filled with platelet lysate. Platelets contain wide range of growth factors and cytokines and can be used as their natural and autologous source. The scaffold was tested in vitro using MG-63 osteoblast-like cells. Cell adhesion, proliferation and osteogenic differentiation were determined. Three different concentrations of liposomes were used and compared to a scaffold with control liposomes filled only with PBS and to the plain polycaprolactone fibers. The results of the study showed that the platelet lysate stimulated cell proliferation. On the other hand, its effect on osteogenic differentiation was not proved. The combination of polymeric fibers with liposomes via simple adhesion was shown to be a promising scaffold for bone tissue engineering applications. In the future, it is necessary to focus on the stability of liposomes to prevent the burst release of the incorporated molecules.
\end{abstract}

\section{INTRODUCTION}

Nowadays 3D materials supporting cell adhesion, infiltration and proliferation are needed in tissue engineering applications. These materials should serve not only as a scaffold, but also promote cell ingrowth and differentiation into the required cell type. Therefore, it is beneficial to combine the scaffolding material with a drug delivery system. Liposomes remain among the nanoparticles most widely used as carriers of active molecules [1]. Nanofibers and microfibers are often used in tissue engineering, because they mimic the natural extracellular matrix (ECM) and enhance cell adhesion and proliferation [2]. Centrifugal spinning uses centrifugal force to produce ultrafine fibers from melts and solutions [3, 4]. This method gives rise to fibers with submicron diameters and fluffy 3D structure. In the current study, PCL fibers prepared using the centrifugal spinning were combined with adhered liposomes. As a natural source of cytokines and growth factors the platelet lysate (PL) was used to boost the growth of MG-63 cells.

\section{Materials AND Methods}

\subsection{Preparation of Fibrous SCAFFOLd WITH ADHERED LIPOSOMES AND ITS CHARACTERIZATION}

Fibrous meshes were prepared using centrifugal spinning device (Cyclone 1000 L/M, FibeRio, USA) from $40 \%$ polycaprolactone (Sigma Aldrich) dissolved in chlorophorm:ethanol in volume ratio 9:1. Orifice G30 and rotation speed 10000 RPM was used. Unilamellar liposomes were prepared from L- $\alpha$-Phosphatidylcholine from egg yolk (SigmaAldrich) using the lipid film hydratation method followed by extrusion (1 $\mu \mathrm{m}$ pore size) [5]. Liposomes were adhered to fibrous scaffolds for 2 hours and subsequently fibers were rinsed with PBS to wash out the non-adhered liposomes. Five different samples were prepared: PCL fibers with maximal concentration of liposomes $(25 \mathrm{mg} / \mathrm{ml}$ ) (PCL/PL1); 4-fold diluted liposomes (PCL/PL2), and 10-fold diluted liposomes (PCL/PL3). Plain PCL fibers (PCL) and PCL fibers with adhered liposomes contained PBS (PCL/PBS) were used as control samples. Fibrous scaffolds with adhered liposomes were visualized using cryo-scanning electron microscopy (FEI Nova NanoSem, CR) and size of liposomes was determined by mastersizer 3000 . Release of growth factors from liposomes with incor- 

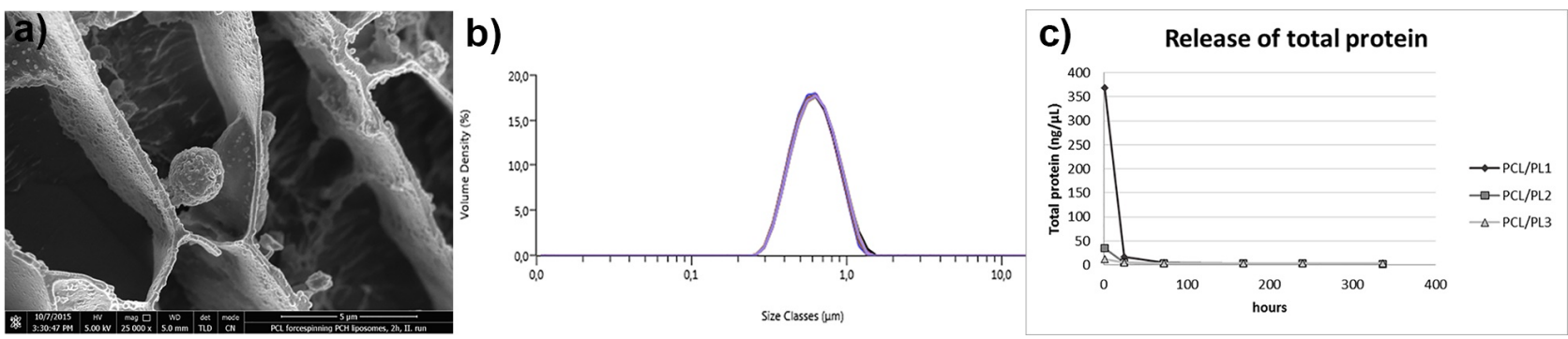

FiguRE 1. Cryo-scanning electron microscopy of fibers prepared by centrifugal spinning techniques with adhered liposomes. Detection of liposomes size distribution using dynamic light scattering (b) and detection of overall protein release (c).
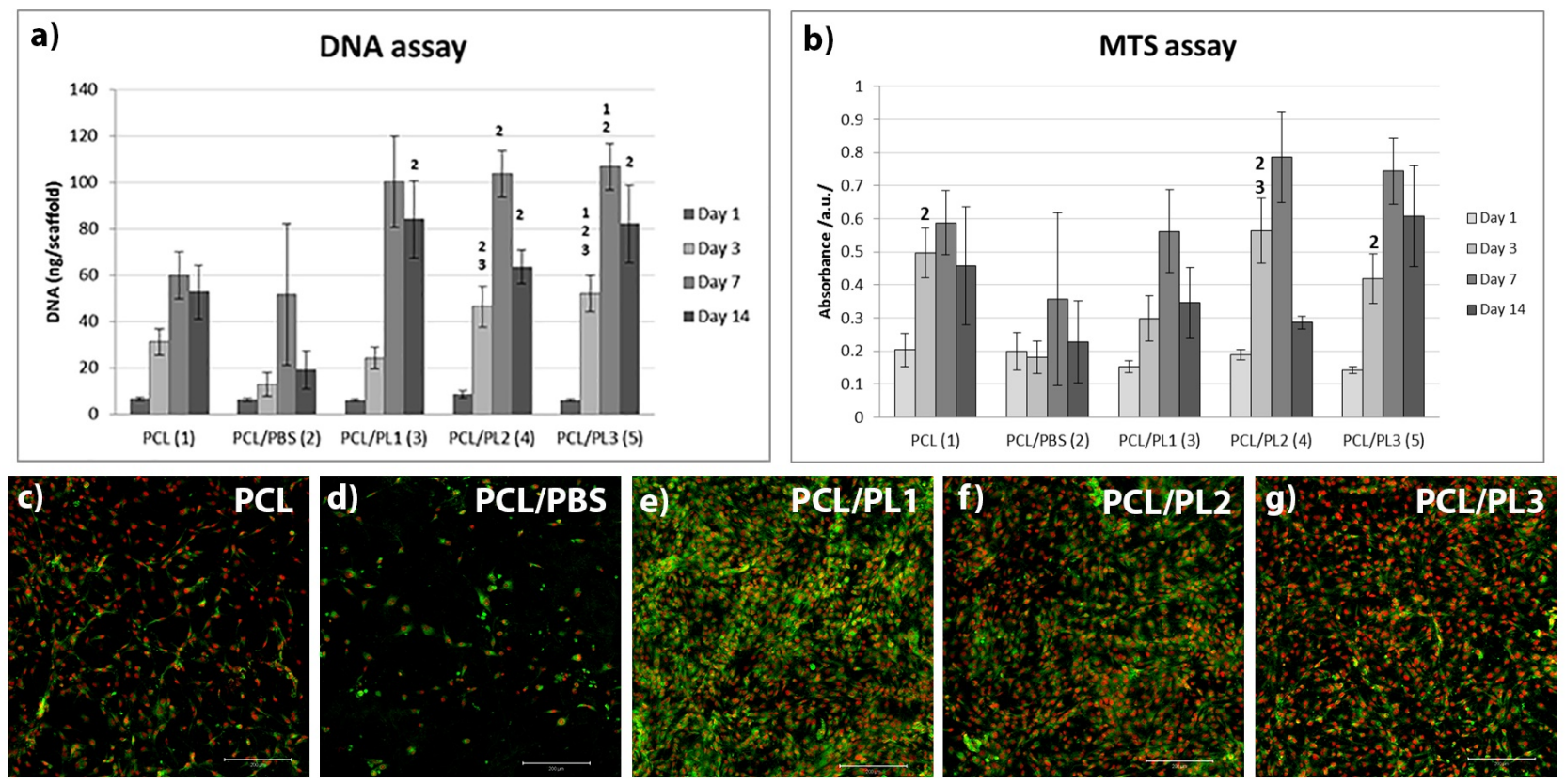

Figure 2. MG-63 adhesion and proliferation on scaffolds measured using DNA quantification (a) and cell metabolic activity measured using MTS test (b). Data were confirmed using confocal microscope. Cell nuclei were stained using PI (red colour) and cell membranes using DiOC6(3) (green). Scale $200 \mu \mathrm{m}$, objective $10 \times$.

porated PL adhered on fibrous scaffolds was measured as a release of overall protein (Quant-iT Protein assay Kit, Life Technologies) and using ELISA (TGF- $\beta 1$, P-Selectin; Duoset®, R\&D Systems, USA).

\subsection{In Vitro test Using MG-63 CELls}

Prepared samples were seeded with $3.1 \times 10^{4} \mathrm{MG}-$ 63 cells $/ \mathrm{cm}^{2}$. The metabolic activity of cells was measured using an MTS assay (CellTiter 96®AQueous One Solution Cell Proliferation Assay; Promega). The proliferation of cells on the scaffold was measured from the amount of DNA (Quant-iT ${ }^{\mathrm{TM}} \mathrm{dsDNA}$ Assay Kit; Life Technologies).

Confocal microscopy (Zeiss LSM DUO) was used to visualize cell adhesion on the scaffolds. Samples were stained using fluorescent probe 3,3'- diethyloxacarbocyanine iodide (DiOC6(3), Invitrogen, $1 \mu \mathrm{g} / \mathrm{mL}$ in $\mathrm{PBS}$ ) and propidium iodide (PI; $5 \mu \mathrm{g} / \mathrm{mL}$ in $\mathrm{PBS}$ ). Osteogenic differentiation was detected as synthesis and production of proteins typical for bone ECM, type I collagen and os- teocalcin using real-time PCR (Light Cycler 480 II; Roche, Switzerland) and immunofluorescence staining (osteocalcin - OCG3, Abcam, UK, AF488 secondary antibody). Results were evaluated statistically using One Way ANOVA.

\section{Results And Discussion}

\subsection{LiPOSOME ADHESION ON FIBERS AND RELEASE OF PROTEIN CONTENT}

Figure 1 shows adhesion of liposomes on the fibers. Liposome size distribution measurement showed that $67 \%$ of liposomes were in range $0.46-0.77 \mu \mathrm{m}$ (Fig 1 $\mathrm{b}$ ). The pattern of release was similar for tested proteins. There was visible burst release from liposomes after the first 15 minutes (Fig 1 c). The strongest release was detected in PCL/PL1 sample with the most liposomes. Small release was visible on PCL/PL1 and PCL/PL2 samples 24 hours after liposome adhesion. The burst release of proteins was caused by low stability of the liposomes. 


\subsection{Cell Viability, Proliferation AND OSTEOGENIC DIFFERENTIATION ON SCAFFOLDS}

Cells adhered similarly on all scaffolds. Cell proliferation was mostly visible on PCL/PL3 and PCL/PL2 respectively. After 14 days of cultivation, DNA amount was higher on all samples containing thrombocyte lysate (PCL/PL1 - 3) compared to the sample with control liposomes with PBS (PCL/PBS) (Fig 2p). Cell viability was comparable for all samples (Fig 2p). Data were confirmed by confocal microscopy. Cells adhered well on all samples and were uniformly distributed. Cells proliferated till day 7 , when confluent layers of cells were visible on PCL/PL1 - 3 (Fig $2 \mathrm{e}-\mathrm{g}$ ). Decrease in cell number was seen on day 14 . Detection of osteogenic markers was very low and did not show any differences between experimental and control samples (data not shown).

Positive influence of PL was shown in following days. Interestingly, positive effect was more visible in samples with 4- and 10-fold diluted liposomes (PCL/PL2 and PCL/PL3) and thus PL Platelets contain a wide spectrum of growth factors and cytokines with different effect. In our study positive effect on cell proliferation was detected. On the other hand, no effect was shown on osteogenic differentiation.

Positive effect of PL was limited by its burst release from the liposomes. Surface adhesion is simple and variable method of functionalization of the surface of fibers. One of the possibilities how to prolong the release is stabilization of liposomes using PEG. Another strategy is to incorporate bioactive molecules inside the fibers. Up to date, only limited number of studies report using of forcespinning fibers as a drug delivery system $[6$.

\section{Conclusion}

Combination of $3 \mathrm{D}$ fibers prepared using centrifugal spinning and liposome adhesion can serve as simple and variable drug delivery system. In the future study we need to focus on liposome stability to prolong the release of incorporated molecules.

\section{ACKNOWLEDGEMENTS}

This research was supported by the Czech Science Foundation Grant No. 15-15697S. Authors thank to dr. Benada from Institute of Microbiology of the CAS for cryo-SEM images.

\section{REFERENCES}

[1] A. Lowery, H. Onishko, D. E. Hallahan, Z. Han. Tumor-targeted delivery of liposome-encapsulated doxorubicin by use of a peptide that selectively binds to irradiated tumors. Journal of Controlled Release 150(1):117 - 124, 2011. DOI:http://dx.doi.org/10.1016/j.jconrel.2010.11.006.

[2] M. Rampichová, J. Chvojka, M. Buzgo, et al. Elastic three-dimensional poly ( $\epsilon$-caprolactone) nanofibre scaffold enhances migration, proliferation and osteogenic differentiation of mesenchymal stem cells. Cell Proliferation 46(1):23-37, 2013. DOI:10.1111/cpr.12001.

[3] A. M. Loordhuswamy, V. R. Krishnaswamy, P. S. Korrapati, et al. Fabrication of highly aligned fibrous scaffolds for tissue regeneration by centrifugal spinning technology. Materials Science and Engineering: $C$ 42:799 - 807, 2014. DOI:http://dx.doi.org/10.1016/j.msec.2014.06.011

[4] M. Rampichová, M. Buzgo, J. Chvojka, et al. Cell penetration to nanofibrous scaffolds: Forcespinning®, an alternative approach for fabricating 3D nanofibers. Cell Adhesion ES Migration 8(1):36 - 41, 2014. DOI:10.4161/cam.27477.

[5] L. D. Mayer, M. B. Bally, M. J. Hope, P. R. Cullis. Techniques for encapsulating bioactive agents into liposomes. Chemistry and Physics of Lipids 40(2):333 345, 1986. DOI:http://dx.doi.org/10.1016/0009-3084(86)90077-0

[6] L. A. Mary, T. Senthilram, S. Suganya, et al. Centrifugal spun ultrafine fibrous web as a potential drug delivery vehicle. eXPRESS Polymer Letters 7(3):238 248, 2013. DOI:10.3144/expresspolymlett.2013.22. 